\title{
Prevention of Thrombo-Embolic Complications in Paraplegics
}

\author{
Professor Dr med. U. F. Gruber ${ }^{1}$ and cand. med. F. Thöni ${ }^{2}$ \\ Department of Surgery, University of Basel, Kantonsspital, CH-4031, Basel, \\ Switzerland
}

\section{Summary}

We have analysed 11 studies dealing with thrombo-embolic complications and their prevention in paraplegics. The incidence of deep vein thrombosis in a total of 1316 hospitalized patients (not receiving any sort of specific prevention), lies between 13 per cent when the diagnosis is made on clinical grounds alone, and reaches 84 per cent when a fibrinogen test and phlebography are carried out routinely. In 904 patients not receiving prevention pulmonary emboli occur in 4-15 per cent, but in only two studies were autopsies carried out. It is most likely that thrombo-embolic complications in paraplegics are even more common than in orthopaedic patients. Five studies dealt with prevention of thrombo-embolic complications; three with oral anticoagulation, two using physical methods, one dealing with subcutaneous heparin and one testing platelet inhibitors. None of the studies published so far is controlled, prospective and randomized at the same time, and uses objective diagnostic methods. Since all studies show one or more methodological problems, it is impossible to make clear statements regarding the relative effectiveness of various prophylactic methods in this type of patient. The apparently extremely high risk for thrombo-embolic complications, however, would make routine prevention in paraplegics mandatory.

\section{Conclusions}

Since thrombo-embolic complications in paraplegics are so frequent, it is of course desirable for the treating physician to institute prevention. Four types of drugs can be considered, the value of all of which has been proven in prospective, controlled and randomized trials. These drugs are Dextran, low dose Heparin, Heparin in combination with dihydro-ergotamine and cumarine derivatives. The main side effect which is mostly feared of all of these drugs is bleeding into the spinal canal. In none of the papers published did this type of complication appear. In none of the papers dealt with here was progressive paralysis due to bleeding recorded. Hachen found bleeding complications in 5.3 per cent, and Silver in 8.8 per cent, but these were mostly gastro-intestinal bleeding, which forced the authors to stop the preventive method.

Correspondence to: Ulrich F. Gruber, Professor of Surgery, Physico-Chemical Toxicology, Kantonsspital, CH-4031, Basel, Switzerland. 